\title{
Comparison of different methods to compute a preliminary orbit of Space Debris using radar observations
}

\author{
Hélène $\mathrm{Ma}^{1}$ and Giovanni F. Gronchi ${ }^{2}$ \\ ${ }^{1}$ Dipartimento di Matematica, Università di Pisa, \\ Largo B. Pontecorvo 5, Pisa, Italy \\ email: helenema@mail.dm.unipi.it \\ ${ }^{2}$ Dipartimento di Matematica, Università di Pisa, \\ Largo B. Pontecorvo 5, Pisa, Italy \\ email: gronchi@dm.unipi.it
}

\begin{abstract}
We advertise a new method of preliminary orbit determination for space debris using radar observations, which we call Infang $\dagger$. We can perform a linkage of two sets of four observations collected at close times. The context is characterized by the accuracy of the range $\rho$, whereas the right ascension $\alpha$ and the declination $\delta$ are much more inaccurate due to observational errors. This method can correct $\alpha, \delta$, assuming the exact knowledge of the range $\rho$. Considering no perturbations from the $J_{2}$ effect, but including errors in the observations, we can compare the new method, the classical method of Gibbs, and the more recent Keplerian integrals method. The development of Infang is still on-going and will be further improved and tested.
\end{abstract}

Keywords. celestial mechanics, preliminary orbit determination, space debris, radar observations, infinitesimal angles.

\section{Introduction}

In the last years, the new method Infang started to be implemented by the University of Pisa and SpaceDys, see Gronchi et al. (2015). We would like to introduce it and compare with two existing methods using data collected from radar observations. We consider two sets of four radar observations to perform a linkage and compute a preliminary orbit. For each set, the times of consecutive observations are very closeł. We denote by $\bar{t}_{j}, j=1,2$ the average epoch for each set.

Radar observations. Each observation is composed by the topocentric distance of the observed object $\rho$, the right ascension $\alpha$ and the declination $\delta$. We assume that $\rho$ is accurate $\boldsymbol{q}$, so that we can obtain a good interpolation of $\dot{\rho}, \ddot{\rho}$. However, the angles are not precisely determined $\|$. An orbit can be expressed in spherical coordinates by the vector $(\alpha, \delta, \dot{\alpha}, \dot{\delta}, \rho, \dot{\rho})$ : therefore here $\dot{\alpha}, \dot{\delta}$ are the unknowns of the preliminary orbit determination problem. This chapter will describe roughly the features of the methods.

$\dagger$ Infang stands for infinitesimal angles

$\ddagger \Delta \mathrm{t}=10 \mathrm{~s}$

q i.e. $\mathrm{RMS} \approx 10 \mathrm{~m}$

$\|$ i.e. $\mathrm{RMS} \approx 0.2^{\circ}$ 


\section{Features of the methods}

Method of Gibbs. It consists in computing the velocity starting from three position vectors at consecutive times $t_{1}<t_{2}<t_{3}$, assuming the observed object follows a Kepler motion. In our case, we can select three observations from the first or the second set.

Method of Keplerian integrals. This method, presented in Taff and Hall (1977) (see also Farnocchia et al. (2010)), uses the orbital elements in spherical coordinates gathered in a radar attributable

$$
\mathcal{A}=(\alpha, \delta, \rho, \dot{\rho})
$$

Instead of $\dot{\alpha}, \dot{\delta}$, we consider as unknowns the quantities

$$
\xi=\rho \dot{\alpha} \cos \delta, \quad \zeta=\rho \dot{\delta},
$$

which are the components of the topocentric velocity in the plane orthogonal to the direction of the line of sight. Assuming the object moves according to the 2-body dynamics, the energy and the angular momentum are conserved, giving a system of linear equations with four unknowns $\left(\xi_{1}, \zeta_{1}, \xi_{2}, \zeta_{2}\right)$, where the indexes refer to epochs $\bar{t}_{1}, \bar{t}_{2}$.

The infinitesimal angles method "Infang". The range $\rho$ is precise, but $\alpha, \delta$ are not accurate. However, the deviations $\Delta \alpha, \Delta \delta$ of the angles from the true values are assumed to be small: therefore they can be treated as "infinitesimal angles". We use the following sets of attributable coordinates:

$$
(\rho, \alpha, \delta, \dot{\rho}, \dot{\alpha}, \dot{\delta})_{j} \text { at } \bar{t}_{j} \text { with } j=1,2 .
$$

This method considers as unknowns $\xi, \zeta, \Delta \alpha, \Delta \delta$ at the two epochs, and uses the equations of motion projected in the direction of the line of sight, the algebraic integrals of Kepler's problem, and Lambert's theorem (see Gronchi et al. (2015) for the details).

Future work. The development of the Infang method is still a work in progress. We intend to investigate the new method with large scale simulations, possibly adding the $J_{2}$ effect.

\section{Acknowledgements}

The work is supported by the Marie Curie Initial Training Network Stardust, FP7PEOPLE-2012-ITN, Grant Agreement 317185.

\section{References}

L. G. Taff, D. L. Hall. 1977, The use of angles and angular rates, I-initial orbit determination, Cel. Mech. ESDyn. Astr., 16, 481488

G. F. Gronchi, L. Dimare, D. Bracali Cioci, H. Ma 2015, in preparation

Herrick, Samuel, Astrodynamics, Vol. I, [2, 7, 8] 1971, London, Van Nostrand Reinhold Co.

D. Farnocchia, G. Tommei, A. Milani, A. Rossi 2010, Innovative methods of Correlation and orbit determination for space debris, Cel. Mech. \& Dyn. Astr., 107 no 1, pp. 169-185. 